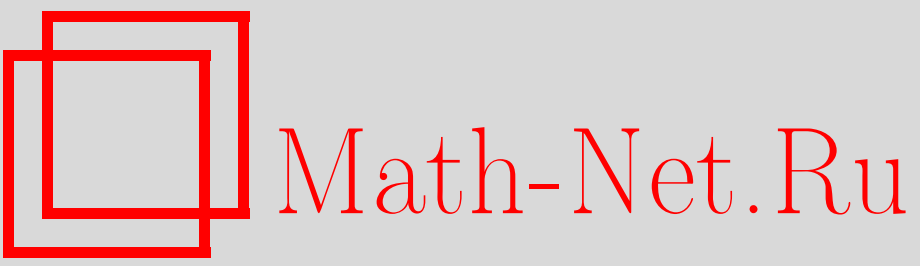

В. Б. Баранецкий, В. П. Котляров, Асимптотическое поведение в области заднего фронта решения уравнения КдФ с начальным условием "типа ступеньки", TMФ, 2001, том 126, номер 2, 214-227

DOI: https://doi.org/10.4213/tmf426

Использование Общероссийского математического портала Math-Net.Ru подразумевает, что вы прочитали и согласны с пользовательским соглашением

http://www. mathnet.ru/rus/agreement

Параметры загрузки:

IP: 54.224 .187 .69

26 апреля 2023 г., 12:59:31 
ТЕОРЕТИЧЕСКАЯ

И МАТЕМАТИЧЕСКАЯ

ФИЗИКА

Том 126, № 2

февраль, 2001

(C) 2001 г. $\quad$ В.Б. Баранецкий* В.П. Котляров*

\section{АСИМПТОТИЧЕСКОЕ ПОВЕДЕНИЕ В ОБЛАСТИ ЗАДНЕГО ФРОНТА РЕШЕНИЯ УРАВНЕНИЯ КДФ С НАЧАЛЬНЫМ УСЛОВИЕМ “ТИПА СТУПЕНЬКИ"}

Получено новое интегральное уравнение, линеаризующее задачу Коши для уравнения Кортевега-де Фриза при начальном условии "типа ступеньки", когда начальная функция исчезает при $x \rightarrow-\infty$ и стремится к некоторой периодической функции при $x \rightarrow+\infty$, и дано разложение решения задачи Коши на радиационную составляющую, определяемую коэффициентом отражения, и составляющую, обусловленную неубывающим характером начального условия. Для второй составляющей решения выведена приближенная детерминантная формула, справедливая при любом $t \geqslant 0$ и $x \in\left(-\infty, X_{N}\right)$, где $X_{N} \rightarrow \infty$ при неограниченном возрастании параметра $N$ конечномерной аппроксимации интегрального уравнения. При $t \rightarrow \infty$ доказано, что решение задачи Коши в окрестности заднего фронта распадается на асимптотические солитоны, фазы которых вычислены явно в терминах коэффициента отражения и других параметров задачи.

\section{1. ВВЕДЕНИЕ}

Задачи об асимптотическом поведении решений нелинейных уравнений типа Кортевега-де Фриза (КдФ) при больших временах остаются актуальными на протяжении всей истории развития теории солитонов. После открытия метода обратной задачи теории рассеяния [1], [2] для интегрирования нелинейных эволюционных уравнений обнаружилась важная роль простейших точных решений этих уравнений, называемых солитонными решениями. В терминах теории рассеяния эти решения характеризуются равенством нулю коэффициента отражения. Так, например, любое начальное возмушение, быстро исчезаюшее при $x \rightarrow \pm \infty$, под воздействием потока, задаваемого каким-либо уравнением типа $\mathrm{K} д \Phi$, распадается при больших временах на солитоны, порожденные дискретным спектром соответствуюшей задачи рассеяния [3]-[5]. Вклад в асимптотику решения, обусловленный непрерывным спектром, т.е. ненулевым коэффициентом отражения, оказывается исчезающе малым при $t \rightarrow \infty[6]-[9]$.

В середине 70-х годов были обнаружены другие интересные объекты теории солитонов - асимптотические солитоны [10]-[12]. В отличие от обычных солитонов они удов-

${ }^{*}$ Институт низких температур им. Б. И. Веркина, Харьков, Украина. E-mail: baranetsky@ilt.kharkov.ua, kotlyarov@ilt.kharkov.ua 
летворяют нелинейному уравнению тем точнее, чем больше $t$. Сушествование таких солитонов было предсказано в работе [13] при изучении уравнения КдФ с начальными данными "типа ступеньки", когда начальная функция исчезает при $x \rightarrow+\infty$ и стремится к некоторой постоянной величине при $x \rightarrow-\infty$. Строгое рассмотрение задачи Коши с начальными данными "типа ступеньки" было проведено в работе [14], где, в частности, было показано, что асимптотические солитоны связаны с однократным непрерывным спектром соответствуюшей задачи рассеяния. Для начальных данных "типа ступеньки", когда начальная функция исчезает при $x \rightarrow+\infty$ и стремится к некоторой периодической или почти периодической функции при $x \rightarrow-\infty$ (см. обзор [12]) были найдены достаточные (близкие к необходимым) условия распада решений на асимптотические солитоны в окрестности переднего фронта, а вопрос о возможности распада решений на асимптотические солитоны в окрестности заднего фронта оставался открытым. После появления работы [15] стало ясно, что такое явление возможно. Описанию распада решений на асимптотические солитоны в окрестности заднего фронта посвяшена эта работа.

Рассмотрим следуюшую задачу Коши для уравнения КдФ:

$$
\begin{gathered}
u_{t}-6 u_{x} u+u_{x x x}=0, \quad x \in \mathbb{R}, \quad t \in \mathbb{R}_{+} \\
u(x, 0)=q(x)
\end{gathered}
$$

где $q(x)$ - достаточно гладкая начальная функция "типа ступеньки", быстро стремящаяся к нулю при $x \rightarrow-\infty$ и к периодической функции $q_{0}(x)$ при $x \rightarrow+\infty$. Для простоты предположим, что функция $q_{0}(x)$ является однозонным потенциалом уравнения Шредингера, а оператор Шредингера

$$
-\frac{d^{2}}{d x^{2}}+q(x), \quad x \in \mathbb{R}
$$

с потеншиалом $q(x)$ не имеет дискретного спектра. При таких предположениях справедливо следуюшее представление для решения задачи Коши (1.1), (1.2):

$$
u(x, t)=-2 \frac{d}{d x} K(x, x, t)
$$

где функция $K(x, y, t)$ является решением линейного интегрального уравнения Марченко

$$
K(x, y, t)+\int_{-\infty}^{x} K(x, z, t) H(z+y, t) d z+H(x+y, t)=0, \quad-\infty<z<x,
$$

с ядром

$$
H(x, t)=\int_{\gamma} p(k) e^{-i k x-8 i k^{3} t} d k+\frac{1}{2 \pi} \int_{-\infty}^{\infty} r(k) e^{-i k x-8 i k^{3} t} d k=P(x, t)+R(x, t)
$$


где $\gamma=[i a, i b]$ - отрезок мнимой оси, $0<a<b$. Здесь функция $r(k)$ является коэффициентом отражения, а функция $i p(i k)$ вешественна в интервале $[a, b]$ и имеет вид

$$
i p(i k)=\sqrt{(k-a)(b-k)} p_{0}(k), \quad k \in[a, b],
$$

где $p_{0}(k)$ - достаточно гладкая и положительная в замкнутом интервале $[a, b](0<a<$ b) функция.

Для исследования распада решения в окрестности заднего фронта уравнение (1.4) неприменимо. Более удобными оказываются другие интегральные уравнения. Будет показано, что решение исходной задачи представимо в виде

$$
u(x, t)=u_{r}(x, t)+2 \frac{d}{d x} \int_{a}^{b} \psi(x, t, k) \hat{h}(x, t, k) \rho(k) d k
$$

где $u_{r}(x, t)=-2 d L(x, x, t) / d x$, а $L(x, y, t)$ есть решение уравнения Марченко

$$
L(x, y, t)+R(x+y, t)+\int_{-\infty}^{x} L(x, z, t) R(y+z, t) d z=0
$$

с ядром $R(x, t)$, определяемым коэффициентом отражения $r(k)$. Вещественная функция $\psi(x, t, k)$ является решением следуюшего основного интегрального уравнения:

$$
\begin{gathered}
\psi(x, t, k)+\int_{a}^{b} A(k, \mu, x, t) \psi(x, t, \mu) \rho(\mu) d \mu=\hat{h}(x, t, k), \quad k \in[a, b] \\
A(k, \mu, x, t)=\int_{-\infty}^{x} \hat{h}(s, t, k) \hat{h}(s, t, \mu) d s .
\end{gathered}
$$

Здесь функции $\rho(\mu)$ и $\hat{h}(x, t, k)$ являются вешественными и определяются формулами

$$
\rho(\mu)=i p(i \mu)>0, \quad \hat{h}(x, t, k)=e^{k x-4 k^{3} t}+\int_{-\infty}^{x} L(x, y, t) e^{k y-4 k^{3} t} d y .
$$

Дальнейшие выкладки будут проводиться для любой достаточно гладкой функции $\rho(\mu)$ такой, что $\rho(\mu)=(\mu-a)^{\alpha}(b-\mu)^{\beta} \rho_{0}(\mu)$, где параметры $\alpha, \beta>-1$, а функция $\rho_{0}(\mu)>0, \mu \in[a, b]$. В этом случае мы получим более широкий класс решений $u(x, t)$ уравнения КдФ, стремящихся к нулю при $x \rightarrow-\infty$ и ограниченных при $x \rightarrow+\infty$.

Для формулировки основных результатов работы введем следующие обозначения. Определим область заднего фронта как множество тех $x \in \mathbb{R}$, для которых

$$
\Omega_{N}(t)=\left\{x \in \mathbb{R} \mid x<4 a^{2} t+\frac{1}{2 a} \ln t^{N+\alpha+1}\right\},
$$

где $N$ - произвольное натуральное число, $t>1$. По известному из решения прямой задачи рассеяния коэффициенту отражения $r(k)$ введем функцию

$$
\eta_{0}(k)=\frac{1}{2 \pi} \int_{-\infty}^{\infty} \frac{\ln \left[1-|r(s)|^{2}\right]}{s^{2}+k^{2}} d s
$$


и определим функцию

$$
D(k, \mu, x, t)=A(k, \mu, x, t) e^{-k\left[x-4 k^{2} t+\eta_{0}(k)\right]} e^{-\mu\left[x-4 \mu^{2} t+\eta_{0}(\mu)\right]}
$$

Обозначим через $D^{(N)}(x, t)=\left\{d_{l m}(x, t)\right\}_{l, m=0}^{N}$ матрицу первых $(N+1)^{2}$ коэффициентов Тейлора функции $D(k, \mu, x, t)$, взятых в точке $k=\mu=a$. Пусть матрица $I^{(N)}(x, t)=$ $\left\{I_{l+m}(x, t)\right\}_{l, m=0}^{N}$ составлена из функций

$$
I_{m}(x, t)=\int_{a}^{b} e^{2 k\left[x-4 k^{2} t+\eta_{0}(k)\right]}(k-a)^{m} \rho(k) d k
$$

а матрица $A^{(N)}(x, t)$ есть произведение матриц:

$$
A^{(N)}(x, t)=D^{(N)}(x, t) I^{(N)}(x, t) .
$$

Предположим также, что $b<2 a$.

Теорема 1. Решение задачи (1.1), (1.2) существует всюду в $\mathbb{R}^{2}$, в области $G_{N}=\bigcup_{t>1} \Omega_{N}(t) \subset \mathbb{R}^{2}$ оно представимо в виде

$$
u(x, t)=u_{r}(x, t)+2 \frac{\partial^{2}}{\partial x \partial d_{00}}\left\{\ln \operatorname{det}\left[E^{(N)}+A^{(N)}(x, t)\right]\right\}+\Delta^{(N)}(x, t)
$$

где $E^{(N)}$ есть единичная матрица порядка $N+1$, функция $u_{r}(x, t)$ определена в (1.6), а функиия $\Delta^{(N)}(x, t)$ удовлетворяет неравенствам

$$
\begin{aligned}
& \left|\Delta^{(N)}(x, t)\right| \leqslant \\
& \leqslant\left\{\begin{array}{l}
\frac{K_{N}}{t}, \\
\frac{N+2+\alpha+\beta}{2 b} \ln \sqrt{\frac{a}{b-a}}<x-4 a^{2} t<\frac{1}{2 a} \ln t^{N+\alpha+1}, \\
\min \left\{\frac{K_{N}}{t}, K\left(\frac{b-a}{a}\right)^{\frac{N+2+\alpha+\beta}{2}} N\right\},-\infty<x-4 a^{2} t<\frac{N+2+\alpha+\beta}{2 b} \ln \sqrt{\frac{a}{b-a} .}
\end{array}\right.
\end{aligned}
$$

Постояннье $K_{N}$ и $K$ зависят от параметров задачи $a, b, \alpha, \beta$ и функиий $r(k) u$ $\rho_{0}(k)$.

Следует отметить, что $\lim _{N \rightarrow \infty} \Delta^{(N)}(x, t)=0$ равномерно по $x \leqslant 4 a^{2} t+((N+2+$

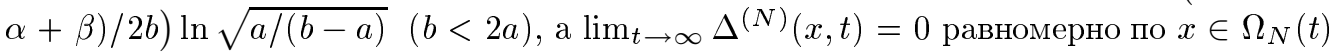
для любого фиксированного натурального $N$. Асимптотический анализ решения (1.12) позволяет доказать, что функция $u(x, t)$ распадается на $[(N+1) / 2]$ солитонов, находяшихся в области $\Omega_{N}(t)$, при $t \rightarrow \infty$. 
Теорема 2. В области $\Omega_{N}(t)$ решение $u(x, t)$ при $t \rightarrow \infty$ представимо в виде

$$
u(x, t)=-\sum_{n=1}^{\left[\frac{N+1}{2}\right]} \frac{2 a^{2}}{\operatorname{ch}^{2}\left\{a\left(x-4 a^{2} t+\frac{1}{2 a} \ln t^{2 n+\alpha-1}+x_{n}\right)\right\}}+O\left(t^{-\frac{1}{4}}\right),
$$

где фазы солитонов имеют вид $x_{n}=x_{n}^{0}+\eta_{0}(a), \eta_{0}(a)$ - значение функиии $\eta_{0}(k)$, определенной в (1.10), взятое в точке $k=a$, а числа

$$
x_{n}^{0}=\frac{1}{2 a} \ln \frac{[(n-1) !]^{2} \Delta_{1}^{(n-1)} \Delta_{2}^{(n-1)} a^{6 n-3+2 \alpha} 2^{10 n-5+4 \alpha}}{\rho_{0}(a)(b-a)^{\beta} \Delta_{1}^{(n)} \Delta_{2}^{(n)}},
$$

где $\Delta_{1}^{(n)}$ и $\Delta_{2}^{(n)}$ - детерминанты матрии, компонентами которых яв.ляются числа $(i+j) ! u \Gamma(i+j+1+\alpha)(i, j=\overline{0, n-1})$, соответственно.

Теорема 2 справедлива и в случае, когда $b>2 a$. Для ее доказательства необходимо провести дополнительные оценки, которые получаются, как и в работе [16], для безотражательного случая.

\section{2. ОСНОВНОЕ ИНТЕГРАЛЬНОЕ УРАВНЕНИЕ}

Рассмотрим уравнение Марченко (1.4) с ядром (1.5). Запишем его в операторной форме

$$
(\mathbf{E}+\mathbf{R}+\mathbf{P})[K]=-R-P
$$

и будем искать решение $K(x, y, t)$ в виде суммы двух функций:

$$
K=L+(\mathbf{E}+\mathbf{R})^{-1}[M]
$$

где первая - $L(x, y, t)$ - удовлетворяет уравнению

$$
(\mathbf{E}+\mathbf{R})[L]=-R,
$$

а вторая есть результат действия оператора $(\mathbf{E}+\mathbf{R})^{-1}$ на некоторую функцию $M(x, y, t)$.

Как известно [17],

$$
(\mathbf{E}+\mathbf{R})^{-1}=\mathbf{E}+\mathbf{Q},
$$

где $\mathbf{Q}$ - интегральный оператор, действуюший в пространстве $L^{1}(-\infty, x)$, и его ядро имеет вид

$$
Q(y, z, t)= \begin{cases}L(y, z, t)+\int_{y}^{x} L(s, y, t) L(s, z, t) d s, & z<y, \\ L(z, y, t)+\int_{z}^{x} L(s, y, t) L(s, z, t) d s, & y<z .\end{cases}
$$

Подстановка (2.2) в (2.1) дает

$$
M+\mathbf{P}(\mathbf{E}+\mathbf{Q})[M]=-P-\mathbf{P}[L] .
$$


Введем в рассмотрение решения Йоста

$$
h(x, t, k)=e^{-i k x}+\int_{-\infty}^{x} L(x, y, t) e^{-i k y} d y
$$

и

$$
\varphi(x, t, k)=e^{-i k x}+\int_{-\infty}^{x} K(x, y, t) e^{-i k y} d y
$$

линейного уравнения Шредингера с потенциалами $u_{r}(x, t)=-2 d L(x, x, t) / d x$ и $u(x, t)=$ $-2 d K(x, x, t) / d x$, соответственно. Эти функции являются аналитическими по переменной $k \in \mathbb{C}_{+}$. Пусть

$$
g(x, z, t, k)=h(z, t, k)+\int_{z}^{x} L(s, z, t) h(s, t, k) d s, \quad k \in \mathbb{C}_{+},
$$

и

$$
F(x, y, z, t)=\int_{\gamma} p(k) e^{-i k y-8 i k^{3} t} g(x, z, t, k) d k .
$$

Тогда из (2.5) следует, что

$$
\mathbf{P}[f]+\mathbf{P Q}[f]=\int_{-\infty}^{x} f(z) F(x, y, z, t) d z,
$$

а правая часть уравнения (2.6) переписывается в виде

$$
-P-\mathbf{P}[L]=-\int_{\gamma} p(k) e^{-i k y-8 i k^{3} t} h(x, t, k) d k=-F(x, y, x, t) .
$$

Исходя из последнего равенства и соотношения (2.9), получаем интегральное уравнение для функции $M(x, y, t)$ :

$$
M(x, y, t)+\int_{-\infty}^{x} F(x, y, z, t) M(x, z, t) d z=-F(x, y, x, t)
$$

С другой стороны, равенство (2.6) дает соотношение

$$
M=-P-\mathbf{P}[K]
$$

которое вместе с (2.8) приводит к следуюшему представлению для функции $M(x, y, t)$ :

$$
M(x, y, t)=-\int_{\gamma} p(k) e^{-i k y-8 i k^{3} t} \varphi(x, t, k) d k .
$$

Поэтому если функция $\varphi(x, t, k)$ есть решение интегрального уравнения

$$
\varphi(x, t, k)-h(x, t, k)+\int_{\gamma} B(x, t, k, \mu) \varphi(x, t, \mu) d \mu=0
$$


с ядром

$$
B(x, t, k, \mu)=p(\mu, t) \int_{-\infty}^{x} h(s, t, k) h(s, t, \mu) d s,
$$

то функция $M(x, y, t)$, определяемая формулой (2.11), будет решением интегрального уравнения (2.10). При этом согласно формулам (1.3), (2.2), (2.11) решение задачи (1.1), (1.2) принимает вид

$$
u(x, t)=u_{r}(x, t)+\frac{1}{\pi} \frac{d}{d x} \int_{\gamma} p(k, t) \varphi(x, t, k) h(x, t, k) d k .
$$

Уравнение (2.12) после замены $k \rightarrow i k, \mu \rightarrow i \mu$ (в результате которой $k, \mu \in[a, b])$ и

$$
\psi(x, t, k)=\varphi(x, t, i k) e^{-4 k^{3} t}, \quad \hat{h}(x, t, k)=h(x, t, i k) e^{-4 k^{3} t}
$$

совпадает с уравнением (1.7) с ядром, определяемым формулами (1.8), (1.9).

\section{3. АНАЛИЗ ОСНОВНОГО ИНТЕГРАЛЬНОГО УРАВНЕНИЯ}

Пусть $L_{\rho}^{2}(a, b)$ есть весовое (с весом $\left.\rho(k)\right)$ пространство вешественных функций $f(k)$, заданных на интервале $(a, b)$, со скалярным произведением

$$
(f, g)_{\rho}=\int_{a}^{b} f(k) g(k) \rho(k) d k .
$$

В этом пространстве рассмотрим оператор $\mathbf{A}$ вида

$$
[\mathbf{A} f](k)=\int_{a}^{b} A(k, \mu, x, t) f(\mu) \rho(\mu) d \mu, \quad k \in(a, b),
$$

зависящий от двух параметров $x, t \in \mathbb{R}$. Ядро этого оператора задано формулой (1.8).

Лемма 1. Оператор $(\mathbf{E}+\mathbf{A})^{-1}$ существует в пространстве $L_{\rho}^{2}(a, b)$, его норма удовлетворяет оценке

$$
\left\|(\mathbf{E}+\mathbf{A})^{-1}\right\| \leqslant 1
$$

для всех $x, t \in \mathbb{R}$.

ДоказАтельство.Для любых $x, t \in \mathbb{R}$ оператор $\mathbf{A}$ определен на всем пространстве $L_{\rho}^{2}(a, b)$, он самосопряжен и вполне непрерывен. Квадратичная форма оператора $\mathbf{A}$ в пространстве $L_{\rho}^{2}(a, b)$ неотрицательна, поскольку в силу (1.8)

$$
(\mathbf{A} f, f)_{\rho}=\int_{-\infty}^{x} d s\left(\int_{a}^{b} \hat{h}(s, t, k) f(k) \rho(k) d k\right)^{2} \geqslant 0 .
$$

Поэтому однородное уравнение

$$
f+\mathbf{A} f=0
$$


имеет только нулевое решение в пространстве $L_{\rho}^{2}(a, b)$. Тогда в силу второй альтернативы Фредгольма неоднородное уравнение

$$
f+\mathbf{A} f=g
$$

однозначно разрешимо в пространстве $L_{\rho}^{2}(a, b)$ при любом $g \in L_{\rho}^{2}(a, b)$. Тем самым оператор $(\mathbf{E}+\mathbf{A})^{-1}$ сушествует и определен на всем пространстве $L_{\rho}^{2}(a, b)$. Оценка для нормы резольвенты в пространстве $L_{\rho}^{2}(a, b)$ следует из неравенств

$$
\|f\|_{\rho}^{2} \leqslant(f, f)_{\rho}+(\mathbf{A} f, f)_{\rho}=(g, f)_{\rho} \leqslant\|g\|_{\rho}\|f\|_{\rho} .
$$

Лемма доказана.

Анализ основного интегрального уравнения построен на аппроксимации этого уравнения интегральным уравнением с вырожденным ядром. Для этого запишем ядро $A(k, \mu$, $x, t$ ) оператора А в виде

$$
A(k, \mu, x, t)=e^{(k+\mu) x-4\left(k^{3}+\mu^{3}\right) t} D(k, \mu, x, t) e^{k \eta_{0}(k)+\mu \eta_{0}(\mu)},
$$

где функция $D(k, \mu, x, t)$ аналитична по $k$ и $\mu$ в области $\{(k, \mu): \operatorname{Re} k>0, \operatorname{Re} \mu>0\}$, гладко зависит от переменных $x$ и $t$ и ограничена по совокупности переменных.

Разложим функцию $D(k, \mu, x, t)$ в окрестности точки $(a, a)$ в ряд Тейлора:

$$
D(k, \mu, x, t)=\sum_{l, m=0}^{\infty} d_{l m}(x, t)(k-a)^{l}(\mu-a)^{m} .
$$

Из неравенств Коши для коэффициентов ряда Тейлора

$$
\left|d_{l m}(x, t)\right| \leqslant a^{-(l+m)} \max _{[a, b] \times[a, b]}|D(k, \mu, x, t)| \leqslant M a^{-(l+m)}
$$

заключаем, что этот ряд сходится абсолютно и равномерно по $(k, \mu) \in(a, b) \times(a, b)$, если $b<2 a$.

Для любого натурального числа $N$ оператор $\mathbf{A}$ можно представить в виде суммы вырож денного оператора $\mathbf{A}_{N}$ и оператора $\mathbf{B}_{N}$, норма которого в пространстве $L_{\rho}^{2}(a, b)$ мала, если $N$ велико. Действительно, положим

$$
\mathbf{A}_{N} f=\sum_{l, m=0}^{N} d_{l m}(x, t) \int_{a}^{b} e^{(k+\mu) x-4\left(k^{3}+\mu^{3}\right) t}(k-a)^{l}(\mu-a)^{m} f(\mu) \rho(\mu) d \mu
$$

и

$$
\mathbf{B}_{N} f=\sum_{(l, m) \in R^{(N)}} d_{l m}(x, t) \int_{a}^{b} e^{(k+\mu) x-4\left(k^{3}+\mu^{3}\right) t}(k-a)^{l}(\mu-a)^{m} f(\mu) \rho(\mu) d \mu,
$$

где в (3.5) суммирование ведется по множеству пар индексов $(l, m)$

$$
R^{(N)}=\{(l, m): 0 \leqslant l, m<\infty\} \backslash\{(l, m): 0 \leqslant l \leqslant N, 0 \leqslant m \leqslant N\} .
$$


ЛЕмма 2. Пусть

$$
x<4 a^{2} t+\frac{N+2+\alpha+\beta}{2 b} \ln \sqrt{\frac{a}{b-a}}, \quad b<2 a, \quad t \geqslant 0 .
$$

Тогда для нормы оператора $\mathbf{B}_{N}$ в пространстве $L_{\rho}^{2}(a, b)$ справедлива оченка

$$
\left\|\mathbf{B}_{N}\right\|_{\rho} \leqslant 2 M \hat{\rho}_{0} a^{1+\alpha+\beta}\left(\frac{a}{2 a-b}\right)^{2}\left(\frac{b-a}{a}\right)^{\frac{N+2+\alpha+\beta}{2}} B(\alpha+1, \beta+1),
$$

əде $\hat{\rho}_{0}=\max _{[a, b]}\left\{\rho_{0}(k)\right\}, \quad M=\max |D(k, \mu, x, t)|$, a $B(\alpha, \beta)-$ функция Эйлера.

ДоКАЗАТЕЛЬСТВО. В силу (3.3) легко видеть, что

$$
\begin{aligned}
& \left|\sum_{(l, m) \in R^{(N)}} d_{l m}(x, t)(k-a)^{l}(\mu-a)^{m}\right| \leqslant \\
& \leqslant M \frac{1}{1-\frac{\mu-a}{a}} \frac{1}{1-\frac{k-a}{a}}\left[\left(\frac{k-a}{a}\right)^{N+1}+\left(\frac{\mu-a}{a}\right)^{N+1}\right] \leqslant \\
& \leqslant 2 M\left(\frac{a}{2 a-b}\right)^{2}\left(\frac{b-a}{a}\right)^{N+1},
\end{aligned}
$$

где в силу предположения, что $b<2 a$, отношение $(b-a) / a$ строго меньше единицы. Тогда для нормы оператора $\mathbf{B}_{N}$ в пространстве $L_{\rho}^{2}(a, b)$ имеем

$$
\left\|\mathbf{B}_{N}\right\|_{\rho}^{2} \leqslant 4 M^{2} \hat{\rho}_{0}^{2}\left(\int_{a}^{b} e^{2 k \xi} e^{-8 k t\left(k^{2}-a^{2}\right)}(b-k)^{\beta}(k-a)^{\alpha} d k\right)^{2}\left(\frac{a}{2 a-b}\right)^{4}\left(\frac{b-a}{a}\right)^{2(N+1)},
$$

где $\xi=x-4 a^{2} t$. Интеграл $J$, входящий в это выражение, легко оценить:

$$
\begin{aligned}
J & \leqslant \int_{a}^{b} e^{2 k \xi}(b-k)^{\beta}(k-a)^{\alpha} d k \leqslant e^{p(\xi)} \int_{a}^{b}(b-k)^{\beta}(k-a)^{\alpha} d k= \\
& =e^{p(\xi)}(b-a)^{1+\alpha+\beta} \int_{0}^{1} s^{\beta}(1-s)^{\alpha} d s=e^{p(\xi)}(b-a)^{1+\alpha+\beta} B(1+\alpha, 1+\beta),
\end{aligned}
$$

где $B(1+\alpha, 1+\beta)$ - функция Эйлера, а $p(\xi)=(b+a) \xi+(b-a)|\xi|$. Поэтому

$$
\left\|\mathbf{B}_{N}\right\|_{\rho} \leqslant 2 M \hat{\rho}_{0}^{1+\alpha+\beta}\left(\frac{a}{2 a-b}\right)^{2} B(1+\alpha, 1+\beta)\left(\frac{b-a}{a}\right)^{N+2+\alpha+\beta} e^{p(\xi)},
$$

и так как для $\xi<((N+2+\alpha+\beta) / 2 b) \ln \sqrt{a /(b-a)}$ выполняется

$$
e^{p(\xi)}<e^{2 b \xi}<\left(\frac{a}{b-a}\right)^{\frac{N+2+\alpha+\beta}{2}}
$$

то получим неравенство (3.6). Лемма доказана. 
ЗАмЕчАниЕ. Лемма 2 показывает, что решение исходной задачи (1.1), (1.2) можно эффективно моделировать при любом $t>0$ в интервале

$$
\left(-\infty, 4 a^{2} t+\frac{N+2+\alpha+\beta}{4 b} \ln \sqrt{\frac{a}{b-a}}\right)
$$

путем решения системы линейных алгебраических уравнений, если произвольно выбранное число $N$ достаточно велико. В случае же, когда $t$ неограниченно возрастает, возникают два дополнительных преимушества этого подхода. Удается, во-первых, построить в замкнутом виде асимптотику решения задачи Коши при $t \rightarrow \infty$ и, во-вторых, расширить область применимости полученных асимптотических формул, так что

$$
x \in\left(-\infty, 4 a^{2} t+\frac{1}{2 a} \ln t^{N+1+\alpha}\right) .
$$

\section{4. АСИМПТОТИЧЕСКИЙ АНАЛИЗ ОСНОВНОГО ИНТЕГРАЛЬНОГО УРАВНЕНИЯ}

Для построения асимптотики ядра (1.8) при $t \rightarrow \infty$ необходимо напомнить асимптотику решения Йоста $h(x, t, i k)(2.7)$ при $k \in[a, b]$. Из равенства (2.3) после применения преобразования Фурье следует, что при $\lambda \in \mathbb{R}$ функция $h(x, t, \lambda)$ удовлетворяет сингулярному интегральному уравнению

$$
\overline{h(x, t, \lambda)}=e^{i \lambda x}+\frac{1}{2 \pi} \int_{-\infty}^{\infty} \frac{r(\mu)}{\mu-\lambda+i 0} e^{-i(\mu-\lambda) x-8 i \mu^{3} t} h(x, t, \mu) d \mu,
$$

а при $\lambda=i k, k \in[a, b]$, функция $h(x, t, \lambda)$ представима в виде

$$
h(x, t, i k)=e^{k x}\left[1+\frac{1}{2 \pi} \int_{-\infty}^{\infty} \frac{r(\mu)}{\mu+i k} e^{-i \mu x-8 i \mu^{3} t} h(x, t, \mu) d \mu\right]
$$

в силу ее аналитичности при $\lambda \in \mathbb{C}_{+}$.

Асимптотика функции $h(x, t, \lambda)$ при $\lambda \in \mathbb{R}$ и $t \rightarrow \infty$ хорошо известна [7], [8]. Следуя [7], найдем, что при $\left|\lambda-k_{s t}^{ \pm}\right|>t^{-1 / 4}\left(k_{s t}^{ \pm}= \pm \sqrt{-X}, X=x / 12 t\right)$ справедливо представление

$$
h(x, t, \lambda)=A(\lambda, X) e^{-i \lambda x}+B(\lambda, X) e^{i \lambda x+8 i \lambda^{3} t}+O\left(t^{-\frac{1}{4}}\right),
$$

где

$$
B(\lambda, X)=-\overline{r(\lambda) A(\lambda, X)} \theta\left(k^{2}+X\right)
$$

и

$$
A(\lambda, X)=\exp \left\{\frac{i}{2 \pi} \int_{-\infty}^{\infty} \frac{\ln \left[1-|r(\mu)|^{2} \theta\left(\mu^{2}+X\right)\right]}{\mu-\lambda-i 0} d \mu\right\} .
$$

Здесь $\theta$ есть функция Хевисайда, т.е. $\theta(k) \equiv 1$ для $k>0$ и $\theta(k) \equiv 0$ для $k<0$. Из ограниченности функции $h(x, t, \lambda)$ при $\lambda \in \mathbb{R}$ и последних формул легко получить, что

$$
h(x, t, i k)=e^{k x}\left[1-\frac{1}{2 \pi} \int_{-\infty}^{\infty} \frac{|r(\mu)|^{2} \overline{A(\mu, X)} \theta\left(\mu^{2}+X\right)}{\mu+i k} d \mu+\delta(x, t, k)\right],
$$


где функция $\delta(x, t, k)$ при $t \rightarrow \infty$ удовлетворяет оценке $|\delta(x, t, k)| \leqslant C t^{-1 / 4}$ для $k \in[a, b]$ и $x \in \mathbb{R}$. Отсюда для функции $\hat{h}(x, t, k)=h(x, t, i k) e^{-4 k^{3} t}$ получаем

$$
\hat{h}(x, t, k)=e^{k\left[x-4 k^{2} t+\eta(k, X)\right]}[1+\hat{\delta}(x, t, k)],
$$

где

$$
\eta(k, X)=\frac{1}{2 \pi} \int_{-\infty}^{\infty} \frac{\ln \left[1-|r(\mu)|^{2} \theta\left(\mu^{2}+X\right)\right]}{\mu^{2}+k^{2}} d \mu,
$$

а функция $r(k)$ - коэффициент отражения. Функция $\hat{\delta}(x, t, k)$ оценивается так же, как и функция $\delta(x, t, k):|\hat{\delta}(x, t, k)| \leqslant C t^{-1 / 4}$. Используя формулу $(1.8)$, окончательно находим, что справедливо равенство

$$
A(k, \mu, x, t)=e^{(k+\mu) x-4\left(k^{3}+\mu^{3}\right) t+k \eta(k, X)+\mu \eta(\mu, X)}\left[\frac{1}{k+\mu}+\Delta(k, \mu, x, t)\right]
$$

с оценкой

$$
|\Delta(k, \mu, x, t)| \leqslant C t^{-\frac{1}{4}}, \quad t \rightarrow \infty .
$$

Эта оценка равномерна по переменным $k, \mu \in[a, b]$ и $x \in \mathbb{R}$.

Лемма 3. Пусть $x \in\left(-\infty, 4 a^{2} t-\sqrt{t}\right)$ u $t \rightarrow \infty$. Тогда решение задачи (1.1), (1.2) имеет вид

$$
u(x, t)=u_{r}(x, t)+O\left(e^{-a \sqrt{t}}\right), \quad t \rightarrow \infty,
$$

где $u_{r}(x, t)=-2 d L(x, x, t) / d x-$ радиачионная составляющая решения, определяемая коэффиииентом отражения $r(k)$.

ЗАмечАниЕ. Асимптотика функции $u_{r}(x, t)$ хорошо известна [6]-[9]. Ее наихудшее убывание по $t$ имеет порядок $O\left(t^{-1 / 2}\right)$.

ДОКАЗАТЕЛЬСТВо ЛЕМмы 3 . Поскольку $\eta(k, X) \leqslant 0$, а $\hat{\delta}(x, t, k)=O\left(t^{-1 / 4}\right)$, то в рассматриваемой области выполняется $|\hat{h}(x, t, k)| \leqslant 2 e^{-a \sqrt{t}}$, если $t$ достаточно велико. Следовательно, справедливы оценки

$$
\|\hat{h}\|_{\rho}^{2} \leqslant 4(b-a) M_{\rho} e^{-2 a \sqrt{t}}, \quad M_{\rho}=\max _{[a, b]} \rho(k)
$$

И

$$
|A(k, \mu, x, t)| \leqslant \frac{2}{a} e^{-2 a \sqrt{t}}
$$

Согласно лемме 1 имеем

$$
\|\psi\|_{\rho} \leqslant\|\hat{h}\|_{\rho} \leqslant 2 e^{-a \sqrt{t}}
$$

Чтобы воспользоваться формулой (1.6), нужно получить оценки для производных по $x$ от функций $\hat{h}(x, t, k)$ и $\psi(x, t, k)$. Для оценки $\hat{h}_{x}^{\prime}(x, t, k)$ воспользуемся уравнением Штурма-Лиувилля и запишем равенство

$$
\hat{h}_{x}^{\prime}(x, t, k)=\int_{-\infty}^{x}\left[k^{2}+u_{r}(y, t)\right] \hat{h}(y, t, k) d y
$$


которое при $t \rightarrow \infty$ влечет неравенство

$$
\left|\hat{h}_{x}^{\prime}(x, t, k)\right| \leqslant 2\left(k+\frac{1}{k}\right) e^{-a \sqrt{t}}
$$

В силу интегрального уравнения (1.7) имеем

$$
\begin{aligned}
\psi_{x}^{\prime}(x, t, k)= & \hat{h}_{x}^{\prime}(x, t, k)-\int_{a}^{b} A(k, \mu, x, t) \psi_{x}^{\prime}(x, t, \mu) \rho(\mu) d \mu- \\
& -\int_{a}^{b} \hat{h}(x, t, k) h(x, t, \mu) \psi(x, t, \mu) \rho(\mu) d \mu
\end{aligned}
$$

Учитывая оценки для функций $\hat{h}(x, t, k), \hat{h}_{x}^{\prime}(x, t, k)$ и $A(k, \mu, x, t)$, полученные выше, из представления (1.6) найдем, что при $t \rightarrow \infty$

$$
u(x, t)-u_{r}(x, t)=O\left(e^{-a \sqrt{t}}\right)
$$

равномерно по $x \in\left(-\infty, 4 a^{2} t-\sqrt{t}\right)$. Лемма доказана.

Перейдем теперь к рассмотрению области тех $x$, для которых

$$
4 a^{2} t-2 \sqrt{t}<x<4 a^{2} t+\frac{1}{2 a} \ln t^{N+1+\alpha} .
$$

Здесь будет доказано, что интегральный оператор $\mathbf{A}(4.2)$ при $t \rightarrow \infty$ представляет собой сумму конечномерного (3.4) и малого (3.5) по норме пространства $L_{\rho}^{2}(a, b)$ операторов. Действительно, для функции $A(k, \mu, x, t)(3.2)$ было доказано, что она при $t \rightarrow \infty$ допускает представление (4.2) с оценкой (4.3). Поэтому коэффициенты $d_{l m}(x, t)$ операторов (3.4) и (3.5) имеют вид

$$
d_{l m}(x, t)=C_{l m}+\Delta_{l m}(x, t)
$$

где числа

$$
C_{l m}=(-1)^{l+m} \frac{(l+m) !}{l ! m !} \frac{1}{(2 a)^{l+m+1}}
$$

являются коэффициентами Тейлора функции $(k+\mu)^{-1}, \mathrm{a}$

$$
\left|\Delta_{l m}(x, t)\right| \leqslant \frac{M}{a^{l+m} t^{\frac{1}{4}}} .
$$

Поскольку в рассматриваемой области $X>0$, то функция $\eta(k, X)(4.1)$ не зависит от $X\left(\theta\left(k^{2}+X\right) \equiv 1\right)$. Тогда $\eta(k, X) \equiv \eta_{0}(k)$ для $X>0$.

Вводя обозначение

$$
E(k, x, t)=e^{k\left[x-4 k^{2} t+\eta_{0}(k)\right]}
$$


и используя неравенство типа (3.7)

$$
\begin{gathered}
\left|\sum_{(l, m) \in R^{(N)}} d_{l m}(x, t)(k-a)^{l}(\mu-a)^{m}\right| \leqslant M(t)\left(\frac{a}{2 a-b}\right)^{2}\left[\left(\frac{k-a}{a}\right)^{N+1}+\left(\frac{\mu-a}{a}\right)^{N+1}\right], \\
\text { где } M(t)=\frac{1}{2 a}+O\left(t^{-\frac{1}{4}}\right),
\end{gathered}
$$

можно записать, что

или иначе

$$
\begin{aligned}
\left\|\mathbf{B}_{N}\right\|_{\rho}^{2} \leqslant & \int_{a}^{b} \int_{a}^{b} E^{2}(k, x, t) E^{2}(\mu, x, t) 2 M^{2}\left(\frac{a}{2 a-b}\right)^{4} \times \\
& \times\left[\left(\frac{k-a}{a}\right)^{2(N+1)}+\left(\frac{\mu-a}{a}\right)^{2(N+1)}\right] \rho(k) \rho(\mu) d k d \mu,
\end{aligned}
$$

$$
\left\|\mathbf{B}_{N}\right\|_{\rho}^{2} \leqslant 4\left(1+\frac{1}{2 a}\right)^{2}\left(\frac{a}{2 a-b}\right)^{4} \frac{1}{a^{2(N+1)}} I_{0}(x, t) I_{2 N+2}(x, t),
$$

где функции $I_{m}(x, t)$ определены в (1.11).

ЛЕмма 4. Интеграль $I_{m}(x, t)$ при $t \rightarrow \infty u \xi=x-4 a^{2} t<\sqrt{t}$ имеют следующее представление:

$$
I_{m}(x, t)=\frac{\rho_{1}}{\left(4 a^{2}\right)^{2(m+\alpha+1)}} \frac{\Gamma(m+\alpha+1)}{t^{m+\alpha+1}} e^{2 a \xi}+\delta_{m}(t, \xi),
$$

где $\rho_{1}=\rho_{0}(a)(b-a)^{\beta} e^{2 a \eta_{0}(a)}$, а $\Gamma(z)-$ гамма-функиия Эйлера. Функиии $\delta_{m}(t, \xi)$ допускают оценки

$$
\left|\delta_{m}(t, \xi)\right| \leqslant \frac{\Gamma(m+\alpha+2) M_{1}^{m+\alpha+2}}{t^{m+\alpha+\frac{3}{2}}} e^{p(\xi)}
$$

с постоянной $M_{1}$, не зависящей от $\xi$, но зависящей от параметров исходной задачи.

Доказательство этой леммы можно найти в [15], [16].

Применяя эту лемму к оценке (4.5) для оператора $\mathbf{B}_{N}$, найдем

$$
\begin{aligned}
\left\|\mathbf{B}_{N}\right\|_{\rho} & \leqslant 2\left(1+\frac{1}{2 a}\right)\left(\frac{a}{2 a-b}\right)^{2} \frac{1}{a^{N+1}} \sqrt{I_{0}(x, t) I_{2 N+2}(x, t)} \leqslant \\
& \leqslant 4\left(1+\frac{1}{2 a}\right)\left(\frac{a}{2 a-b}\right)^{2} \frac{1}{a^{N+1}} \frac{\rho_{1} e^{2 a \xi}}{t^{N+\alpha+2}} \frac{\sqrt{\Gamma(\alpha+1) \Gamma(2 N+\alpha+3)}}{(4 a)^{2 N+2 \alpha+4}} \leqslant K_{N},
\end{aligned}
$$

если $2 a \xi<\ln t^{N+\alpha+1}$. Таким образом, норма оператора $\mathbf{B}_{N}$ в пространстве $L_{\rho}^{2}(a, b)$ мала при $t \rightarrow \infty$. Конечномерньй оператор $\mathbf{A}_{N}(3.4)$ отличается от соответствуюшего вырожденного оператора $\mathbf{A}_{N}$ из работ [15], [16] тем, что коэффициенты $C_{l m}$ следует заменить на $C_{l m}+\Delta_{l m}(x, t)$, где для функций $\Delta_{l m}(x, t)$ справедлива оценка (4.4), а весовая функция $\rho(k)$ приобретает дополнительный множитель $e^{2 k \eta_{0}(k)}$, т.е. $\hat{\rho}(k)=$ $\rho(k) e^{2 k \eta_{0}(k)}$. Поэтому завершение доказательств теорем 1 и 2 почти не отличается от приведенного в работе [16].

Наличие экспоненциального множителя $e^{2 k \eta_{0}(k)}$ у весовой функции $\hat{\rho}(k)$ приводит к дополнительному, в сравнении с безотражательным случаем, сдвигу фаз асимптотических солитонов, обусловленных ненулевым коэффициентом отражения. 


\section{Список литературы}

[1] C.S. Gardner, J. M. Green, M.D. Kruskal, R. M. Miura. Phys. Rev. Lett. 1967. V. 19. P. 1095.

[2] В. Е. Захаров, А. Б. Шабат. ЖЭТФ. 1971. Т. 61. С. 118.

[3] А. Б. Шабат. ДАН СССР. 1973. Т. 211. С. 1310.

[4] Л.А. Тахтаджкян, Л.Д. Фаддеев. Гамильтонов подход в теории солитонов. М.: Наука, 1986.

[5] M. Ablowitz, H. Segur. Solitons and the Inverse Scattering Transform. Philadelphia: SIAM, 1981.

[6] В. Е. Захаров, С. В. Манаков. ЖЭТФ. 1976. Т. 71. С. 203.

[7] В.Е. Захаров, С.В. Манаков, С.П. Новиков, Л.П. Питаевский. Теория солитонов: Метод обратной задачи. М.: Наука, 1980.

[8] В. С. Буслаев, В. В. Суханов. Зап. научн. семин. ЛОМИ. 1982. Т. 120. С. 31; 1982. Т. 138. С. 8; Пробл. мат. физ. 1982. Т. 10. С. $70 ; 1986$. Т. 11. С. 78.

[9] P. Deift, X. Zhou. Asymptotics for the Painlevé II equation: Announcement and results. In: Spectral Scattering Theory and Applications. Proc. of a Conference on Spectral and Scattering Theory Held at Tokyo Institute of Technology (June 30-July 3, 1992) Adv. Stud. Pure Math. V. 23. Ed. K. Yajima. Tokyo: Kinokuniya Company Ltd, 1994. P. 17.

[10] Е. Я. Хруслов. Письма в ЖЭТФ. 1975. Т. 21. № 8. С. 469.

[11] В. П. Котляров, Е.Я. Хруслов. ТМФ. 1986. Т. 68. № 2. С. 172.

[12] E. Ya. Khruslov, V.P. Kotlyarov. Soliton asymptotics of nondecreasing solutions of nonlinear completely integrable evolution equations. In: Spectral Operator Theory and Related Topics. Adv. Sov. Math. V. 19. Ed. V. A. Marchenko. Providence, RI: AMS, 1994. P. 129.

[13] А. В. Гуревич, Л. П. Питаевский. Письма в ЖЭТФ. 1973. Т. 17. № 5. С. 268.

[14] Е. Я. Хруслов. Матем. сб. 1976. Т. 99. № 2. С. 261.

[15] E. Ya. Khruslov, S. Holger. Мат. физика, анализ, геометрия. 1998. № 1/2. С. 49.

[16] В. Б. Баранецкий. Мат. физика, анализ, геометрия. 1999. № 3/4. С. 199.

[17] В. А. Марченко. Операторы Штурма-Лиувилля и их приложения. Киев: Наукова думка, 1977. 